Complementary

Medicine Research

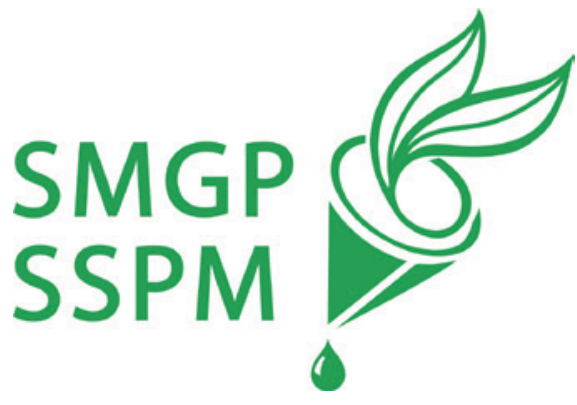

Korrespondenzadresse Geschäftsstelle SMGP

Dr. Franz Huber

c/o Zürcher Hochschule für angewandte Wissenschaften

Life Sciences and Facility Management

Grüental, Postfach, 8820 Wädenswil, Schweiz

f.huber@smgp.ch

www.smgp.ch

\section{Society Bulletins / Gesellschaftsmitteilungen}

Complement Med Res 2021;28:85-86

DOI: $10.1159 / 000514424$

\section{Joyeux Noël et}

\section{Bonne Année 2021!}

Encore une année passée, mais quelle année! Qui aurait pensé il y a douze mois en arrière, à travers quelles épreuves l'humanité allait passer en 2020 ? La pandémie a bousculé notre vie émotionnelle, personnelle et professionnelle. La SSPM a également dû s'adapter face à cette pandémie : Nous avons dû annuler, repousser et réorganiser nos cours.

Cette année, seuls le cours 6 du 3 mars 2020 sur les maladies de l'appareil urogénital et la sortie botanique du 29 août ont pu se dérouler comme prévu.

Lors de l'excursion, malgré un temps très pluvieux et peu accueillant, nous avons pu profiter de la beauté du paysage au Pont du Nant et découvrir le Jardin botanique alpin " La Thomasia » avec sa richesse en plantes alpines. Une bouffée d'air frais entre deux vagues de restrictions...

L'assemblée générale ainsi que le cours de base ont dû être repoussés à l'année prochaine. Prévu initialement fin mai 2020 à Leysin, le cours de base est maintenant planifié pour fin mai 2021 à Montézillon dans le canton de Neuchâtel, et nous espérons que la situation pandémique se sera améliorée d'ici-là.

En raison de la deuxième vague et des mesures prises par l'Université de Lausanne, le cours 10 (La Phytothérapie dans le domaine de la médecine complémentaire) ainsi que le cours 7 (Phytothérapie lors de maladies psychiques, et sédatives à base de plantes) ont dû être organisés sous forme de Webinaire. Malgré le manque de contact personnel et l'absence d'échanges, ces cours ont eu un succès réjouissant.
Avec plus de 40 participants, le cours 7 a même battu le record de participation pour la Suisse romande ! La forme et les modalités des cours ont l'air de plaire car les évaluations et commentaires sont, à notre grande satisfaction, très positifs.

Notre prochain évènement (cours 3 « Phytothérapie et système cardiovasculaire » en février 2021) est à nouveau planifié sous forme de Webinaire. Cependant, pour la suite, nous espérons vivement pouvoir organiser les cours suivants, dont le cours de base, sous forme présentielle. Le programme de l'année 2021 est dorénavant en ligne et vous pouvez le visualiser ici.

Un autre changement a marqué l'année 2020 au sein de la SSPM /SMGP. Après de nombreuses années riches et couronnées de succès, le Prof. Dr. Beat Meier s'est retiré du poste de secrétaire général de la SSPM/SMGP, et le Dr. Franz Huber a repris sa succession à partir du ler juillet 2020. La SSPM remercie chaleureusement le Prof. Dr. Beat Meier pour son engagement sans faille dans le domaine de la Phytothérapie tout au long de ces années et souhaite au Dr. Franz Huber un bon début dans ses nouvelles fonctions.

Il nous reste à remercier tous ceux qui ont soutenu et continuent à soutenir inlassablement la phytothérapie et notre association. Nous espérons vous revoir l'année prochaine lors de nos évènements et vous souhaitons de tout cœur de rester en bonne santé et de joyeuses fêtes de fin d'année avec vos proches!

Barbara Zürcher,

Présidente Section romande SSPM

Karoline Fotinos-Graf,

Secrétariat SSPM/Coordination cours 


\section{SSPM Cours 10 et 7 par webinaire : Mission accomplie !}

À la suite des circonstances spéciales liées au Covid-19 les deux derniers cours SSPM du 17 septembre 2020 et du 19 novembre 2020 ont eu lieu sous forme virtuelle : Une première au sein de la SSPM. Ce nouveau mode de cours a suscité beaucoup d'intérêt parmi nos membres et d'autres intéressés : Jamais auparavant autant de participants ne s'étaient inscrits à un cours SSPM, un nouveau record !

La préparation a été plutôt intensive, autant pour les conférenciers que pour les organisatrices : Les conférenciers ont dû enregistrer leurs conférences au préalable à la maison - quelques problèmes techniques survenant pendant les enregistrements ont heureusement pu être résolus - et pour finir il fallait convertir toutes les conférences en vidéos. Mais, tout est bien qui finit bien : Les liens pour visionner les vidéos ont pu être transmis aux participants, qui avaient pour certains pris congé afin de regarder les vidéos lors de la journée " officielle " du cours. D'autres ont préféré profiter de cette nouvelle flexibilité afin de consulter les vidéos à un autre moment, par exemple le soir ou le week-end. Les QCM demandés - servant comme "preuve de présence "nous ont été retournés, et après contrôle les attestations de participation ont pu être émises.

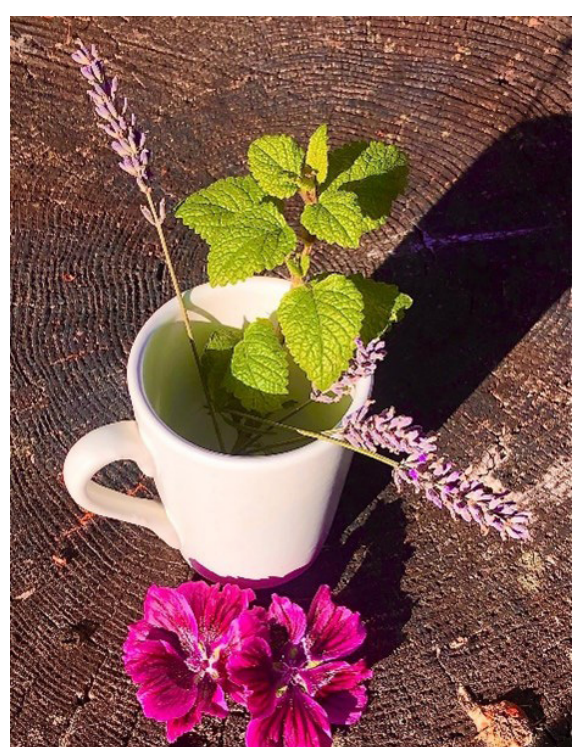

Lors du cours 10, avec le thème " La Phytothérapie dans l'environnement de la médecine complémentaire ", le Professeur Hostettmann a tout d'abord donné un survol sur les plantes médicinales enregistrées en tant que phytomédicaments et les plantes utilisées traditionnellement chez nous. Le Dr Jaggi, médecin en anthroposophie, nous a donné un aperçu des particularités de la médecine anthroposophique, avec son principe de la salutogénèse. La Dre Cornaz, médecin en MTC, nous a parlé des 5 éléments selon lesquels chacune des 13000 plantes utilisées médicalement est classée. Le Dr Tauxe nous a parlé des différentes méthodes de préparation des plantes, et nous a parlé plus en détail de la préparation spagyrique. Christine Funke nous a présenté d'autres aspects de la spagyrie, ainsi que la gemmothérapie, la thérapie par les bourgeons. Pour finir Karoline FotinosGraf a guidé les participants au travers du labyrinthe des réglementations et de la problématique de la délimitation, notamment entre phytomédicaments et compléments alimentaires.

Lors du cours 7 « Phytothérapie lors de maladies psychiques, et sédatifs à base de plantes » le Dr Pamplona nous a présenté les plantes à utiliser lors de troubles du sommeil et d'anxiété. Le Dr Tauxe nous a parlé de la prise en charge de l'anxiété et du stress par l'aromathérapie, avec la présentation de quelques cas pratiques. La Dre Corcelle a expliqué le syndrome dépressif et sa prise en charge du point de vue de la micronutrition ainsi que de la phytothérapie. Un survol sur les plantes pouvant être appliquées lors d'un déclin des fonctions cognitives a été donné par le Professeur Hostettmann, et Karoline Fotinos-Graf a traité plus en détail une des plantes mentionnées, le Ginkgo biloba. Les pratiques et usages du cannabis médical, plante souvent mentionnée par les patients pour traiter leurs troubles du sommeil (CBD), ont été présentés par la Dr Eigenmann. Pour finir Karoline Fotinos-Graf nous a présenté quelques options phytothérapeutiques pour traiter certains cas d'hyperactivité (TDA/H).

Les retours et évaluations des participants étaient très positives, le seul petit bémol - prévisible - étant le manque de contact direct tant pour discuter avec d'autres participants que pour poser les questions. Les échanges professionnels et personnels faisant partie intégrante de nos cours, nous espérons que la situation de pandémie s'améliorera peu à peu pour pouvoir reprendre les cours sous leur forme habituelle.

\section{Formation continue 2021}

La formation continue en phytothérapie est destinée principalement aux médecins et aux pharmaciens et se déroule sur trois ans sous forme de 4 modules, avec 3-4 cours par année, des excursions, des congrès, ainsi qu'un travail de certificat. Ce système est flexible, ce qui vous permet de débuter cette formation à tout moment

Pour les médecins, la formation postgraduée et continue se termine par un certificat reconnu par la FMH intitulé "Certificat de capacité en phytothérapie SSPM». Les médecins qui auront obtenus ce titre, peuvent le faire reconnaître auprès des caisses-maladies par le système Tarmed, sous la position " prestation en phytothérapie " depuis le 1er janvier 2018. Pour les pharmaciens, il y a la possibilité de faire valoir ce titre par la FPH comme "Certificat complémentaire FPH en phytothérapie». Les autres professions médicales obtiennent le titre de «Certificat en phytothérapie SSPM».

Vous trouverez les détails de cette formation continue ainsi que les règlements correspondants sous le lien www.sspm.org.

\section{Crédits}

- SGAIM et autres sociétés de discipline médicale: Reconnus dans le cadre de la formation élargie

- FPH: 50 points pour un jour de formation - 25 points pour la journée d'excursion

- Sociétés professionnelles selon les directives ou les accréditations par la SSPM 